2012

\title{
Does Race and National Origin Influence the Hourly Wages That Latino Males Receive?
}

Clara E. Rodriguez

Fordham University, crodriguez@fordham.edu

Grigoris Argeros

Fordham University

Michael Hajime Miyawaki

Fordham University

Follow this and additional works at: https://fordham.bepress.com/soc_facultypubs

Part of the Demography, Population, and Ecology Commons, Inequality and Stratification Commons, Politics and Social Change Commons, Race and Ethnicity Commons, and the Work, Economy and Organizations Commons

\section{Recommended Citation}

Rodriguez, Clara E.; Argeros, Grigoris; and Miyawaki, Michael Hajime, "Does Race and National Origin Influence the Hourly Wages That Latino Males Receive?" (2012). Sociology Faculty Publications. 16.

https://fordham.bepress.com/soc_facultypubs/16 


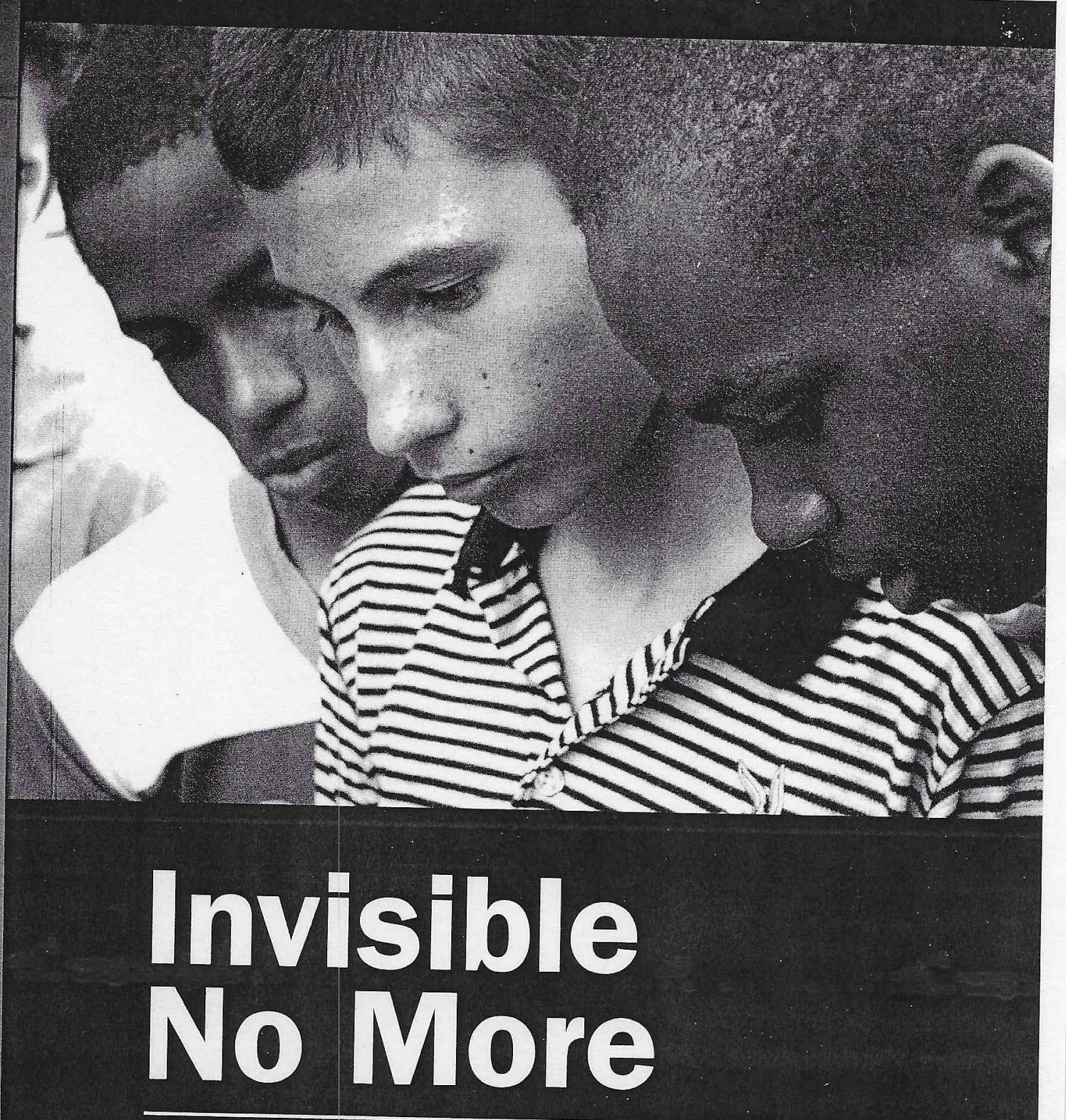

Understanding the Disenfranchisement of Latino Men and Boys

Edited by Pedro Noguera, Aída Hurtado, and Edward Fergus 


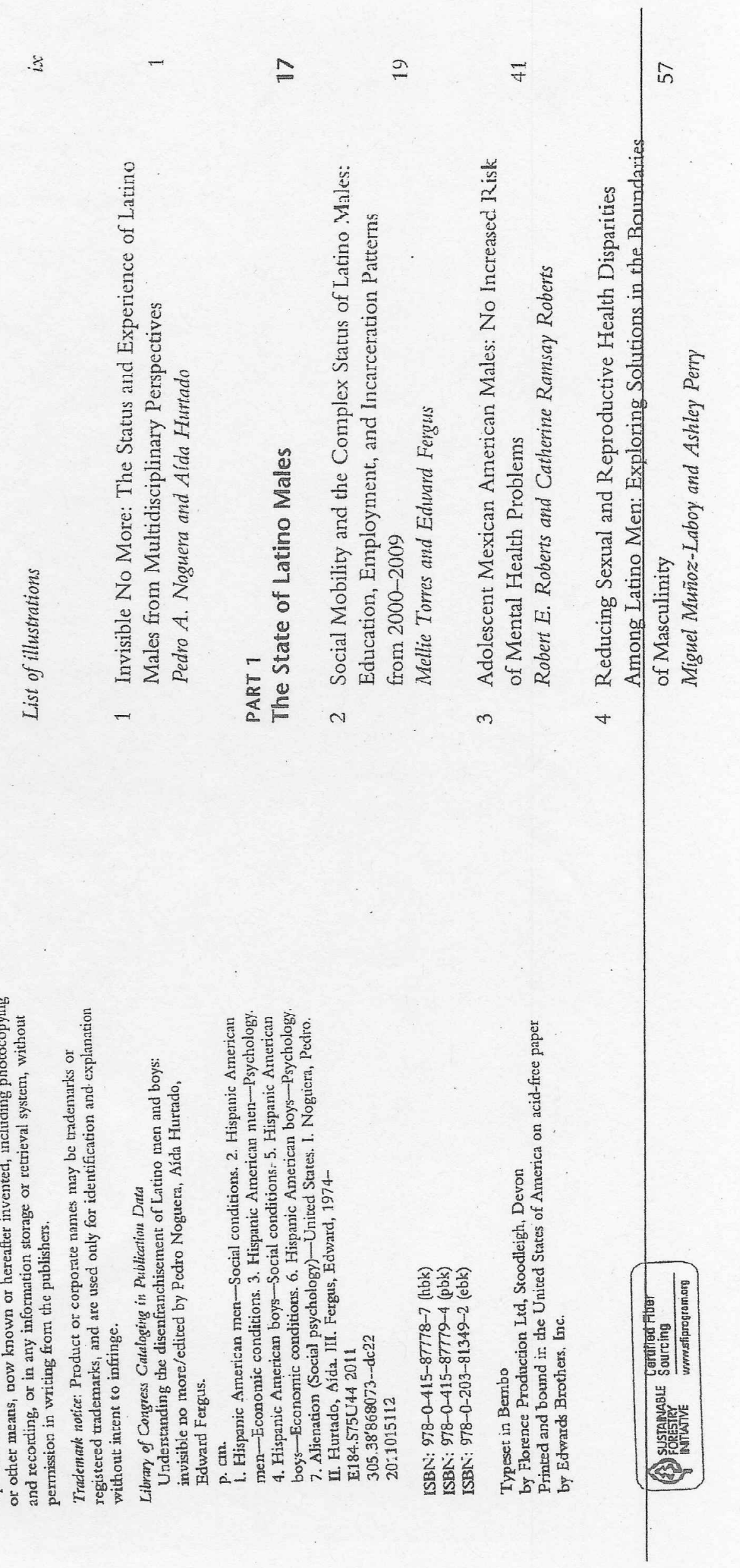




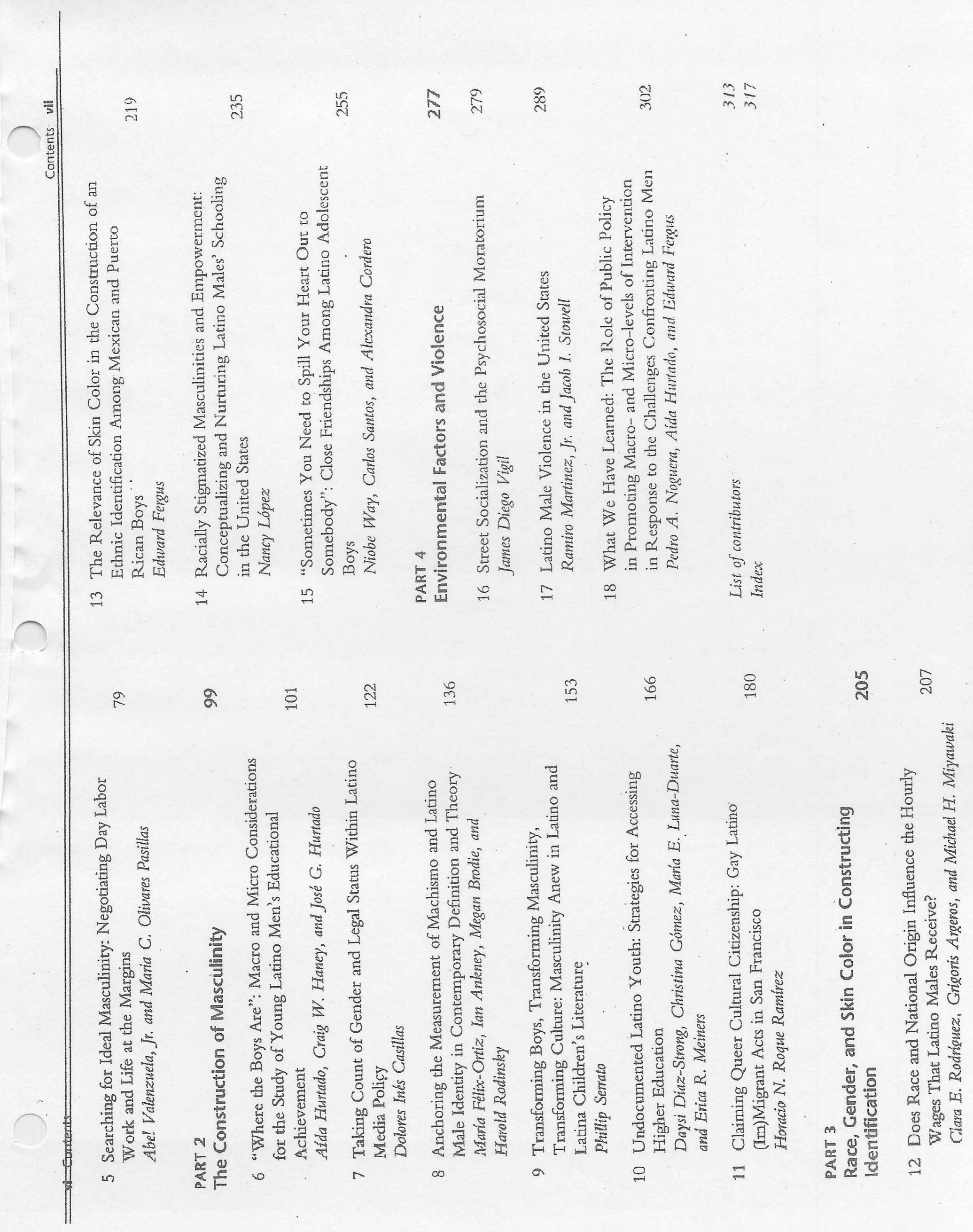




\section{INVISIBLE NO MORE}

Latino men and boys in the United States are confronted with a wide variety of hardships that are not easily explained or understood. They are populating prisons, dropping out of high school, and over-represented in the service industry at alarming degrees. Young Latino men, especially, have among the lowest wages earned in the country, a rapidly growing rate of HIV/AIDS, and one of the highest mortality rates due to homicide. Although there has been growing interest in the status of men in American society, there is a glaring lack of research and scholarly
work available on Latino men and boys.

This groundbreaking interdisciplinary volume, edited by renowned scholars Pedro Noguera, Aída Hurtado and Edward Fergus, addresses the dearth of scholarship and information about Latino men and boys to further our understanding of the unique challenges and obstacles that they confront during this health, criminal justice, education, represent a cross section of disciplines from sociology and more. By drawing attention to psychology, economics, labor, segment of the population, this volume to the sweeping issues facing this principles and overarching guidelines for decreasing the invisibility and a set of disenfranchisement of Latino men and boys.

Pedro Noguera is Peter L. Agnew Professor of Education in the Steinhardt School of Culture, Education, and Human Development at New York
University.

Aída Hurtado is Professor and Luis Leal Endowed Chair in the Chicana and Chicano Studies Department at the University of California, Santa Barbara.

Edward Fergus is Deputy Director of the Metropolitan Center for Urban Education at New York University. 


\section{INVISIBLE NO MORE}

Understanding the

Disenfranchisement of

Latino Men and Boys

Edited by

Pedro Noguera

Aída Hurtado

Edward Fergus 


\title{
12 \\ DOES RACE AND NATIONAL ORIGIN INFLUENCE THE HOURLY WAGES THAT LATINO MALES RECEIVE?
}

\author{
Clara E. Rodríguez \\ Grigoris Argeros \\ Michael H. Miyawaki
}

In the process of writing this chapter, law SB 1070 was passed by the state of Arizona, which requires that police determine the immigrant status of people they stop and suspect are in the country illegally. Some referred to this as sanctioning
racial profiling. The passage of new attention to the way individuas raised the importance of "race" and brought people in positions of political or hiring are identified by others, in particular by others can substantially impact whaw people are identified by labor market. In the case of Latinos, happens to them in everyday life and in the of race, skin color, and phenotype one is extensive literature on the impact outcomes (see, for example, Arce et al their life chances and socioeconomic Espino \& Franz, 2002; Frank et al., 2010; G7; Cotton, 1993; Darity et al., 2002; \& Telles, 1996; Rodríguez, 1990, 1991; Gomez, 2000; Logan, 2004; Murguia It is, however, only recently that this arena, where it has been tied to the conse has moved to a more public and political Indeed, replicating the results of earlier sted issue of undocumented immigration. (2010) study of immigrants who were granted on all Latinos, Frank and colleagues found that darker-skinned counterparts. reported race of Additionally, this study investigates the infled to the hourly wages they receive. wages of Latino males. We examine influence of national origin on the hourly intersectionality of race, national these questions within the context of the attainment, English language proficigins, and other variables (i.e., educational 


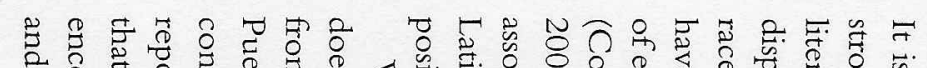

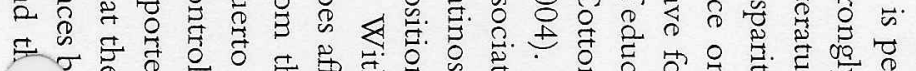

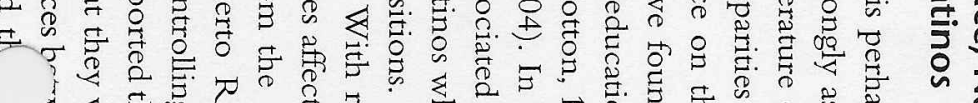

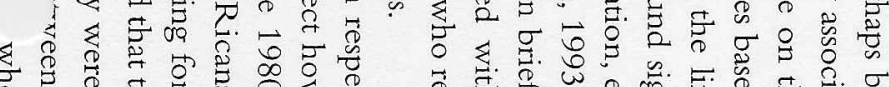

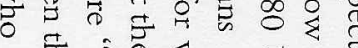

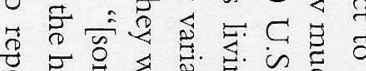

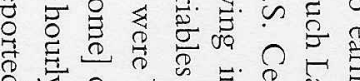

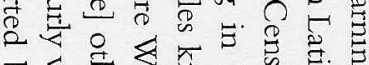

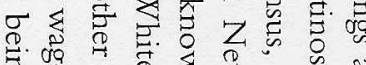

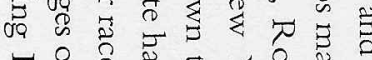

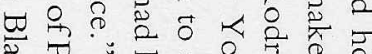

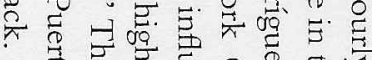

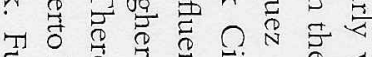

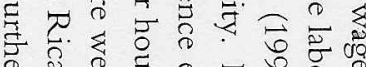

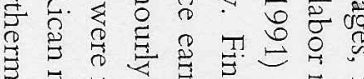

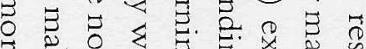

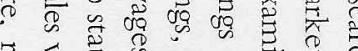

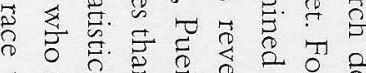

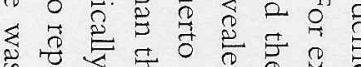

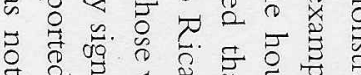
क

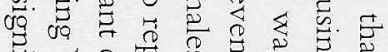

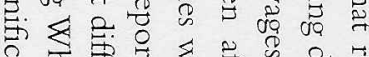

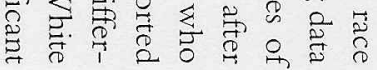

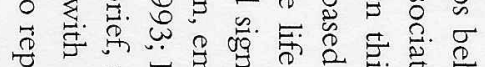
2 $\begin{aligned} & 0 \\ & 0\end{aligned}$

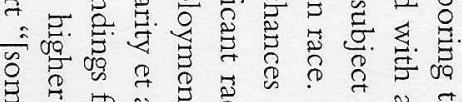

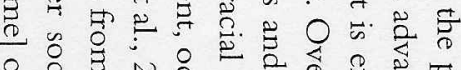

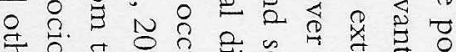

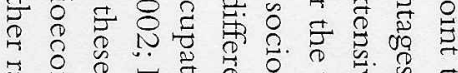

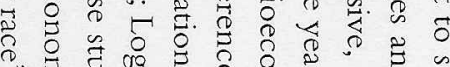

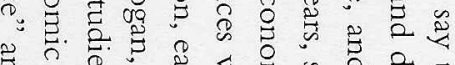
(

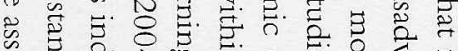

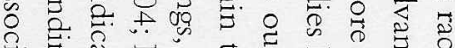

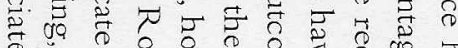

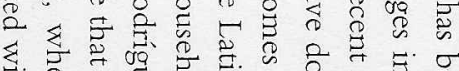

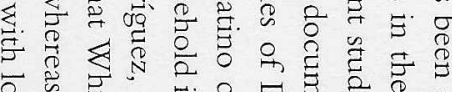

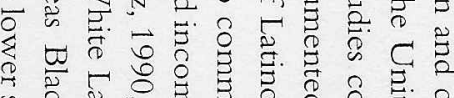

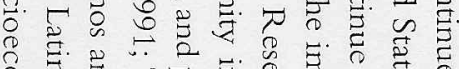

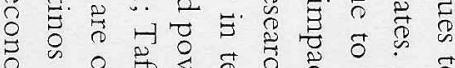

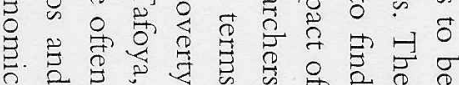

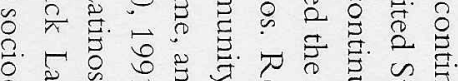

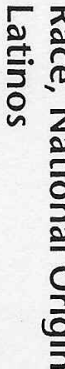

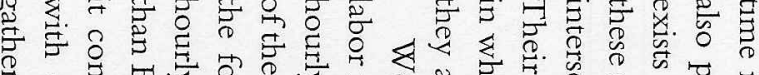

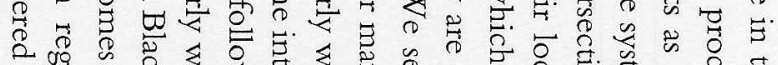

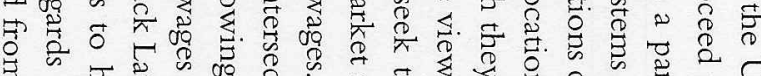

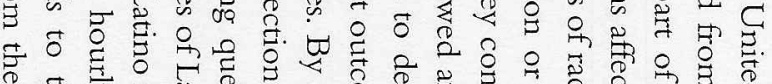

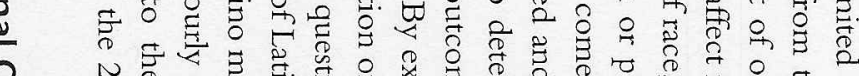

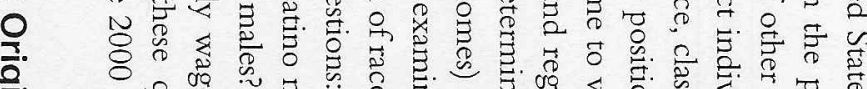

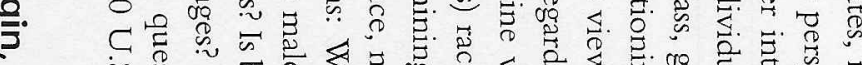

\% की

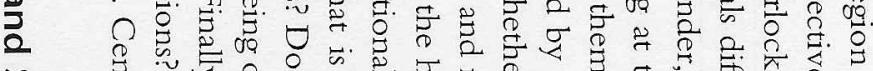

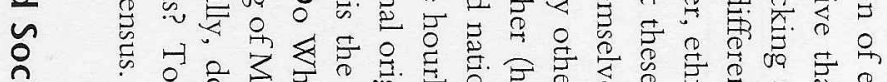

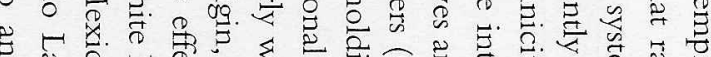

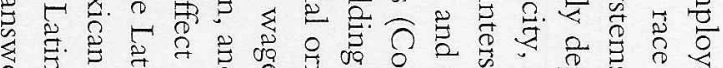

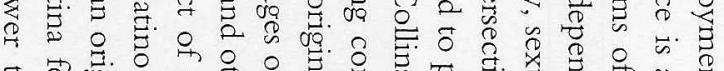

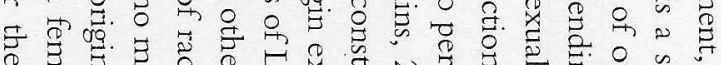

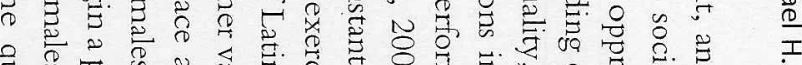

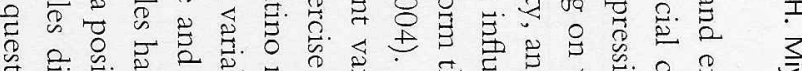

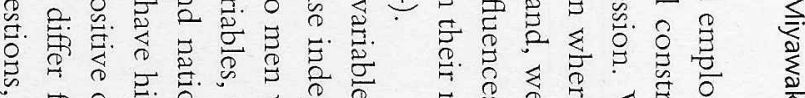
क웅 뭉

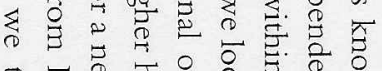

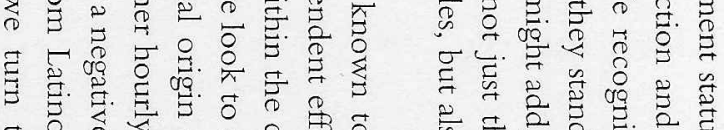

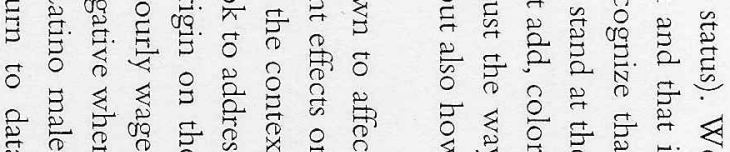

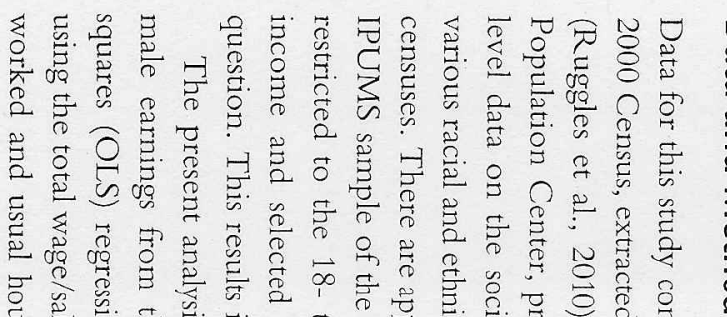

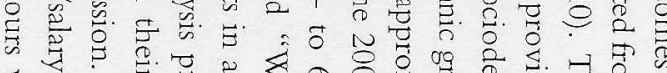
\&

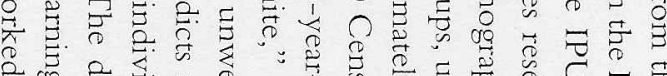

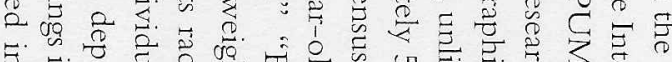

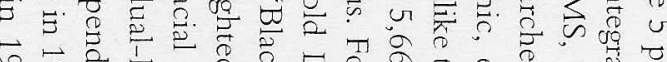
b :

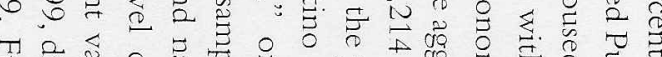
E

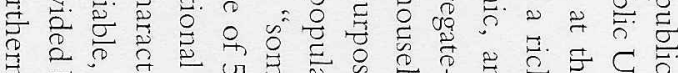

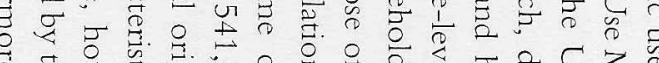

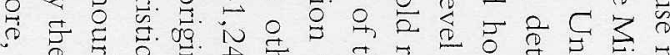

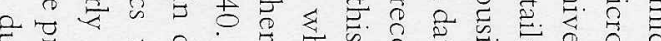

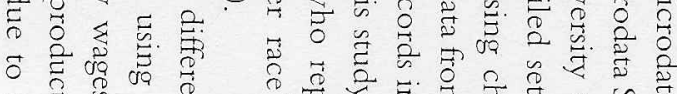

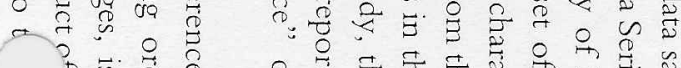

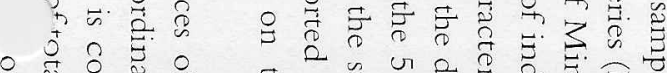

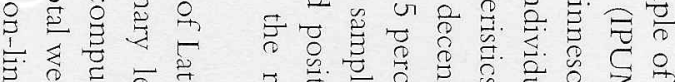

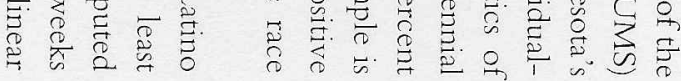

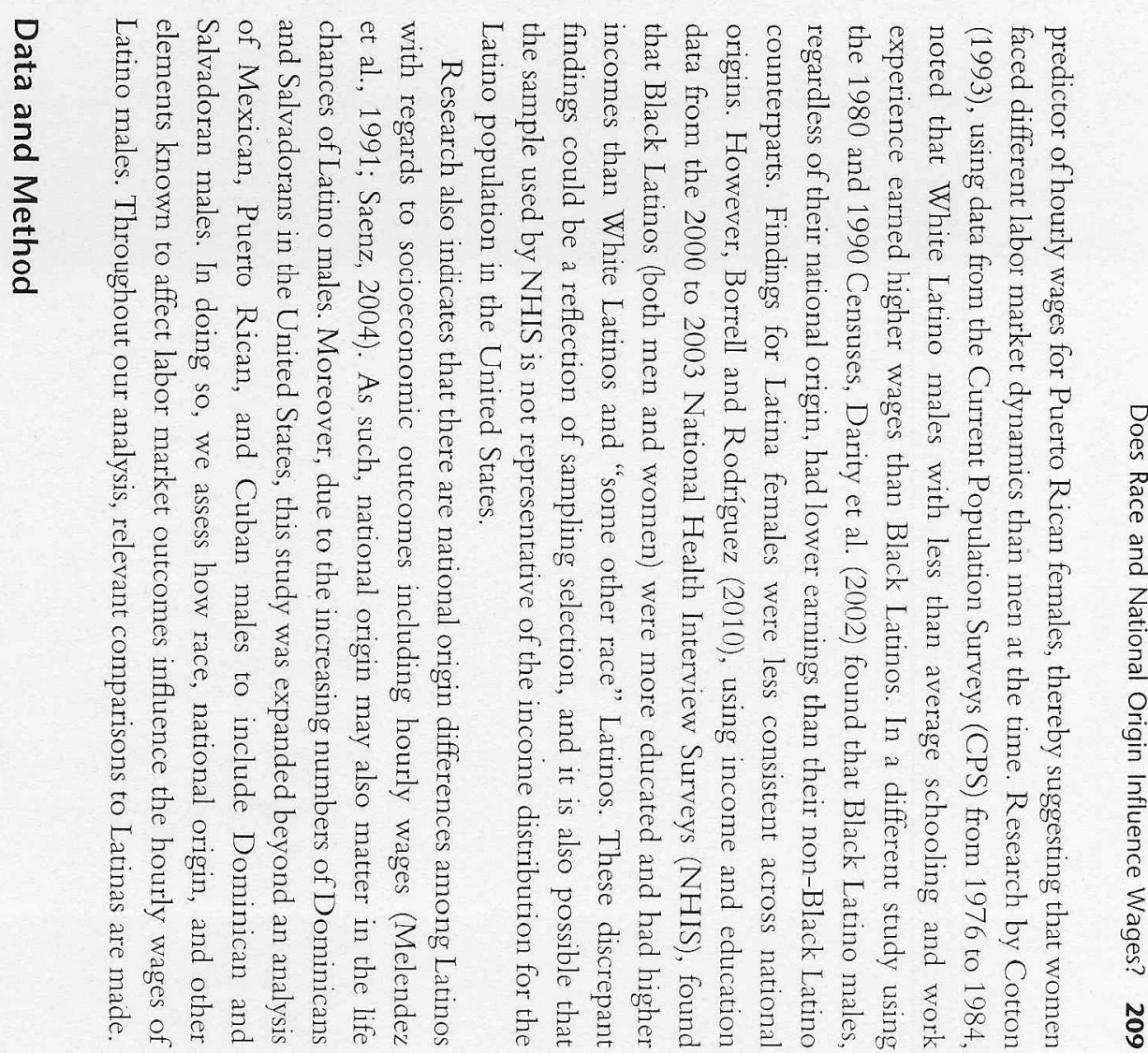




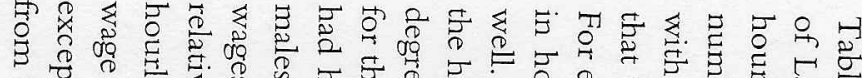

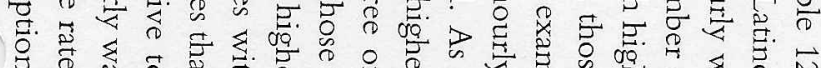

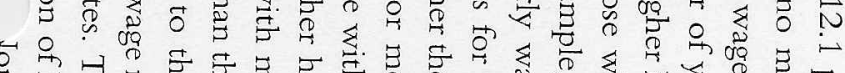

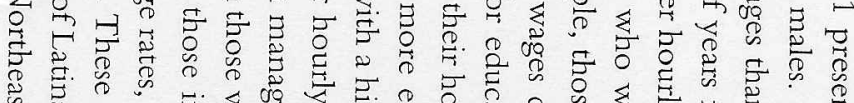

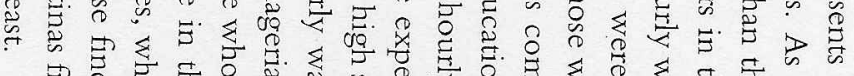

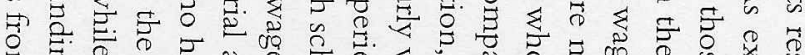

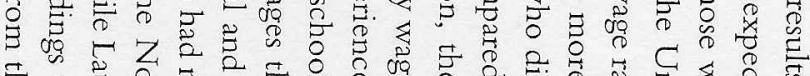

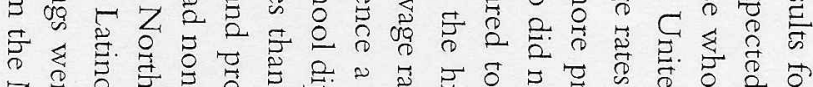
3 万人

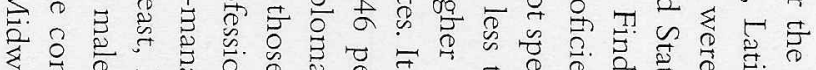

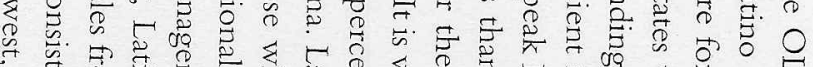

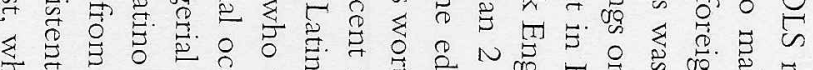

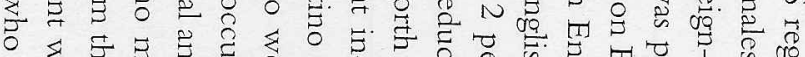

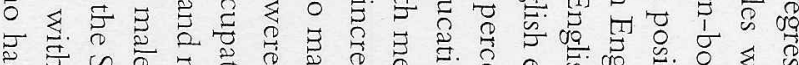

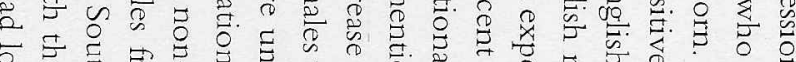

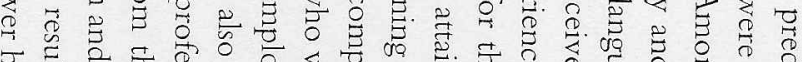

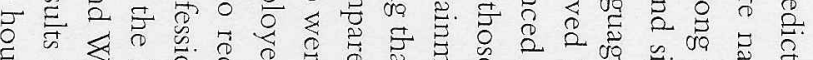

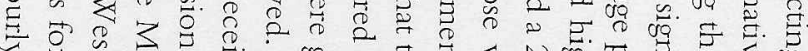

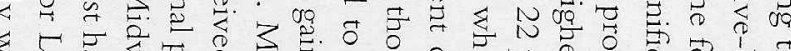

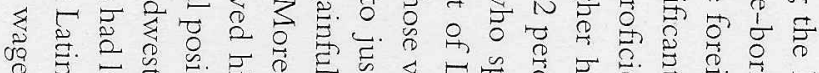

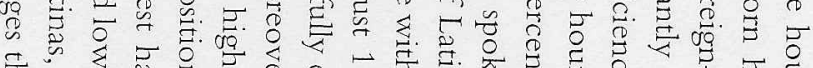

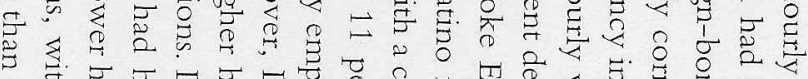

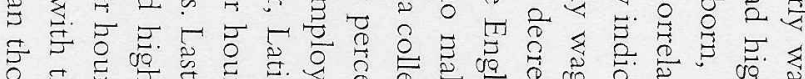

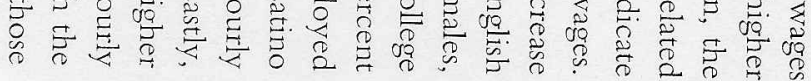

$\stackrel{\substack{D \\ \stackrel{n}{n}}}{\vec{n}}$

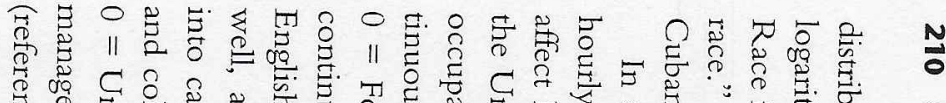

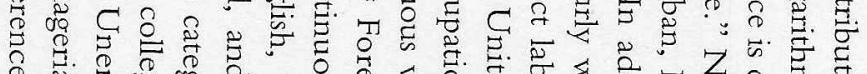

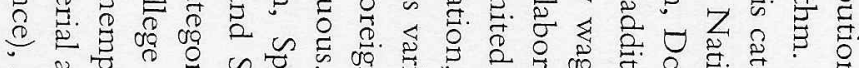

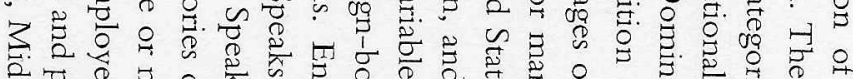

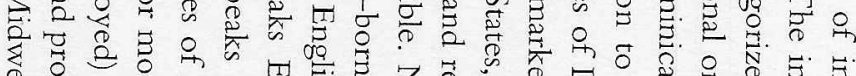

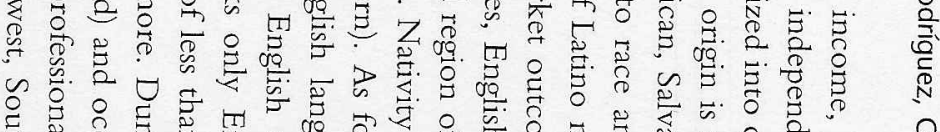

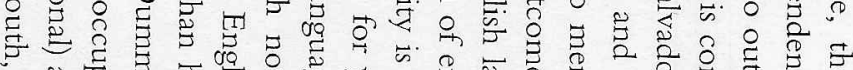

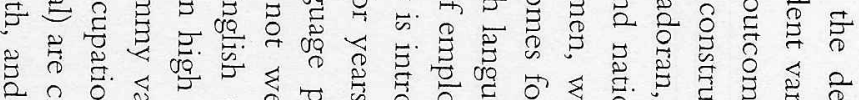

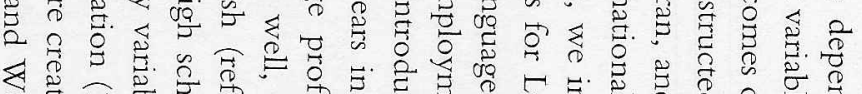

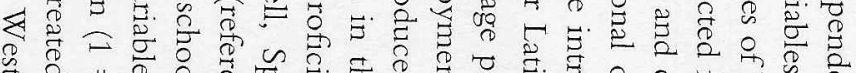

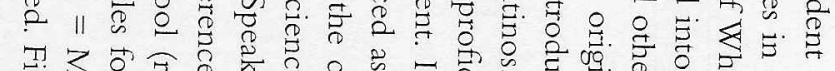

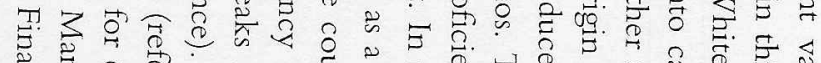

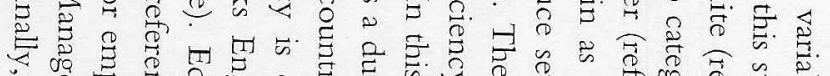

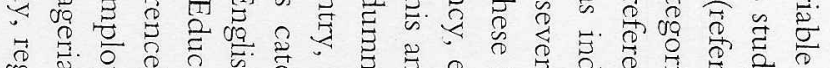

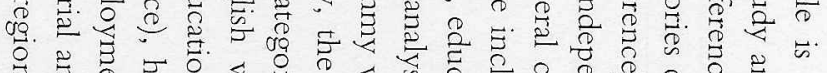

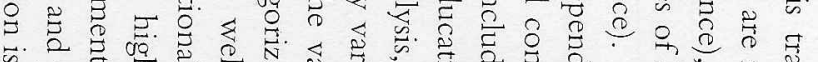

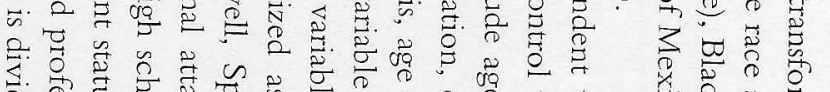
है

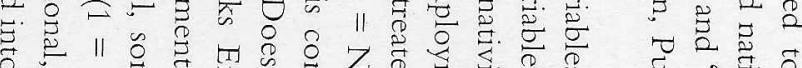

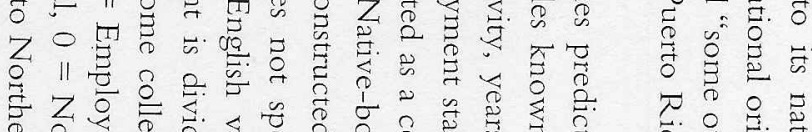

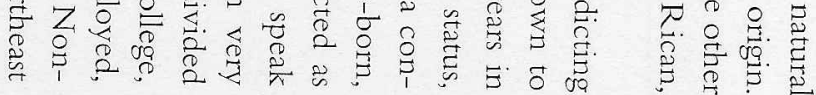

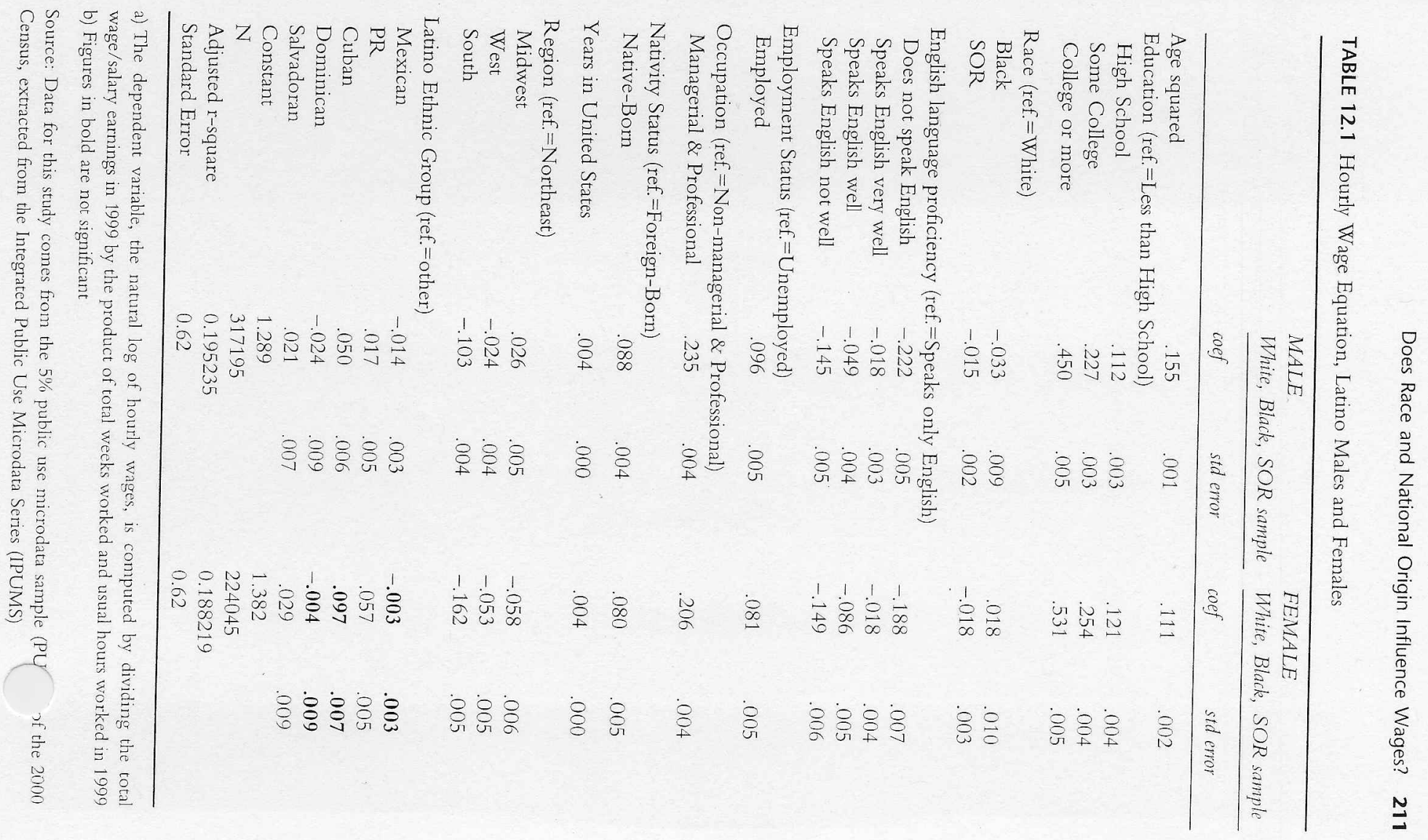




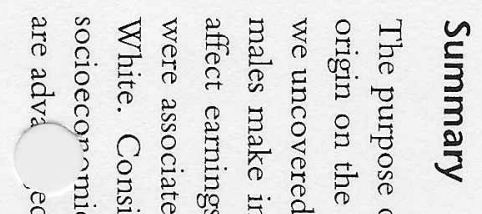

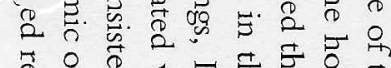

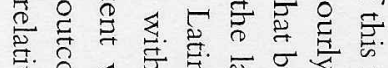

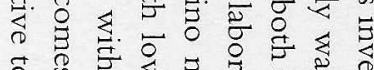
के

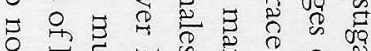
들 \&.

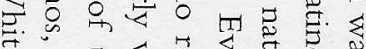

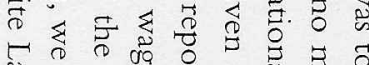
. 远

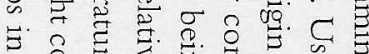

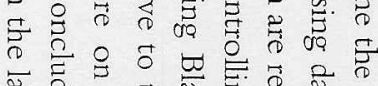

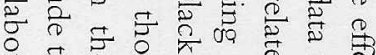

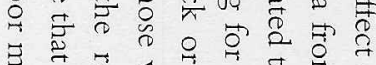

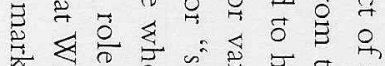

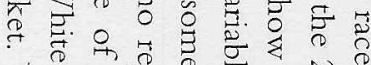

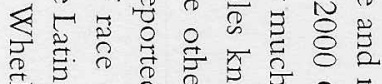
90

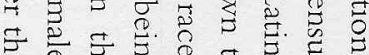

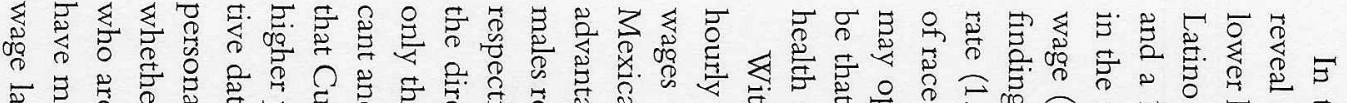

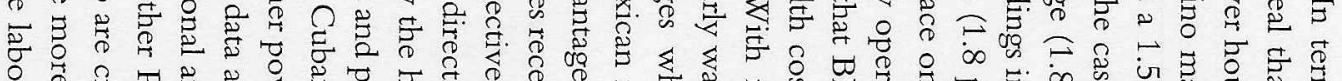

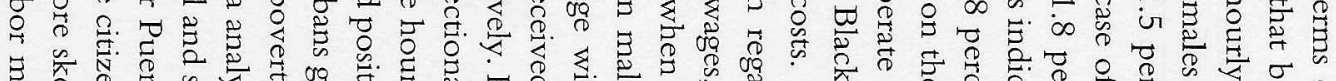

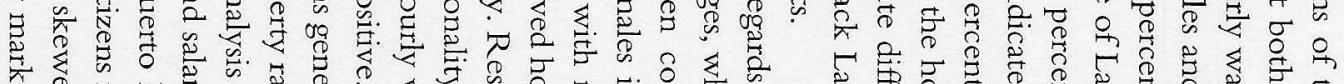

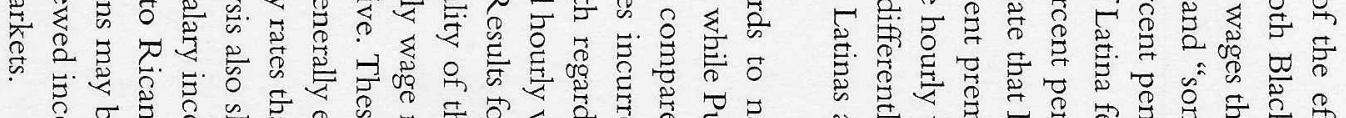

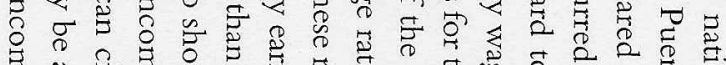

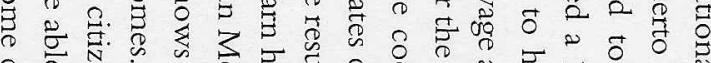

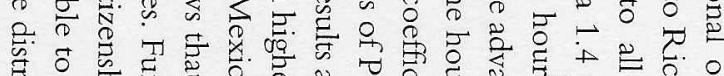

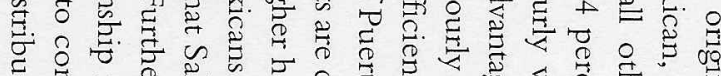

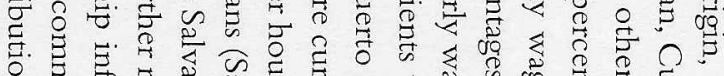

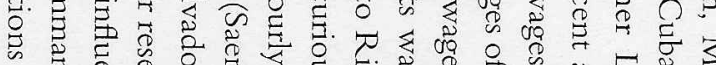

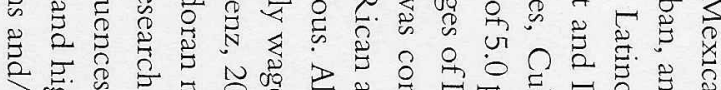

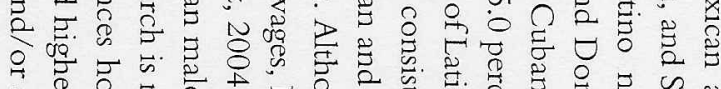

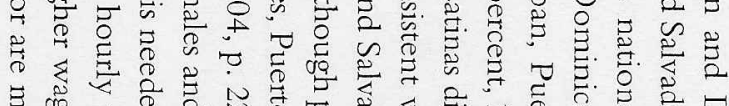

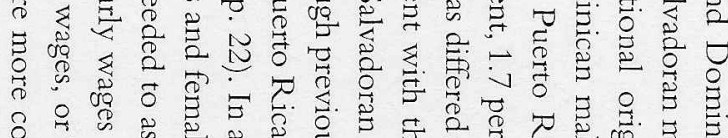

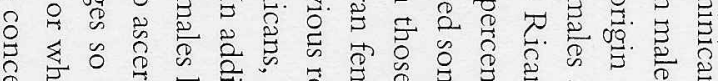

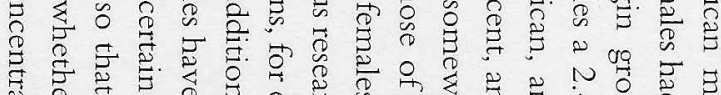

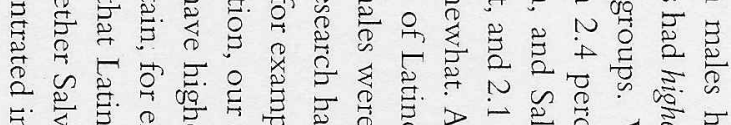

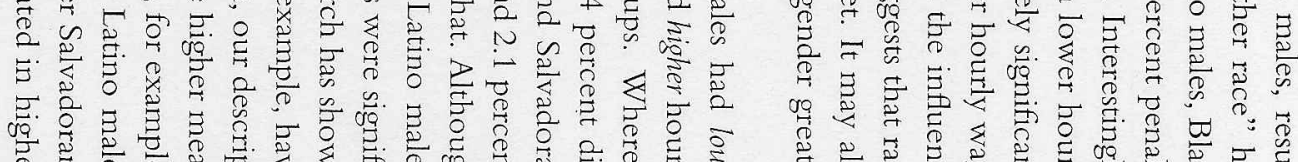

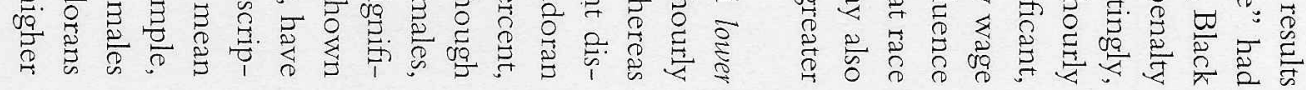

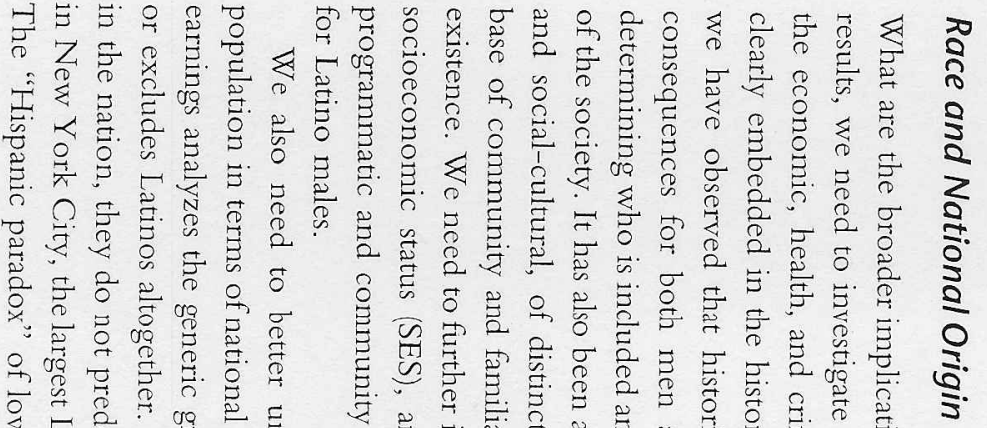

政 包商: 藏

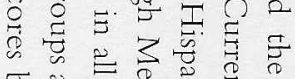

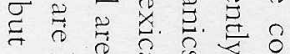
و छ है

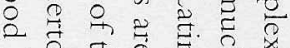

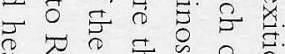

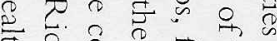
छ

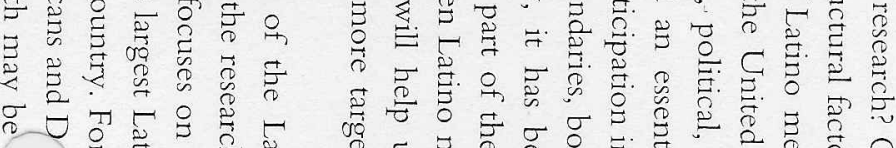
$\because \stackrel{\Xi}{\Xi} \mathfrak{\Xi}$

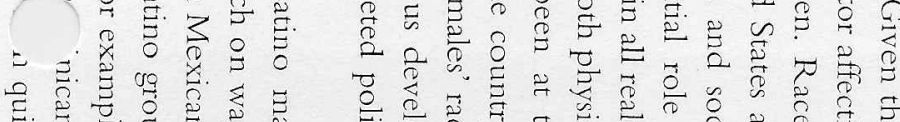

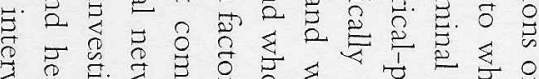
孚

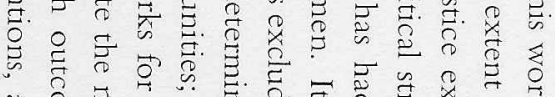

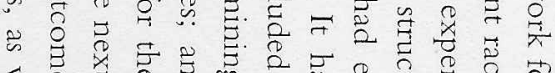
¿

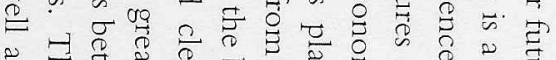
↔ Е. ¿

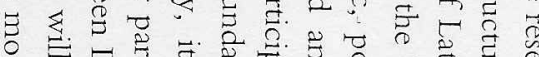

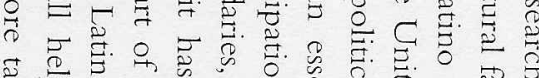

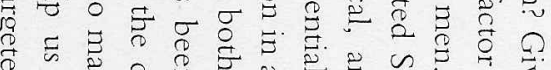

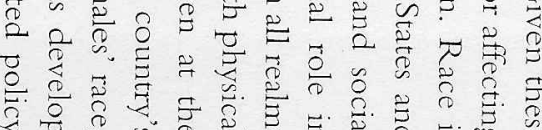

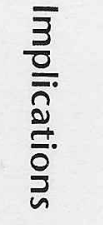

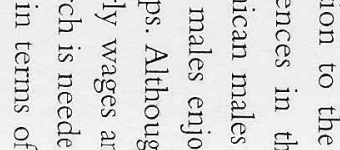

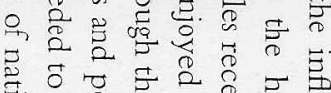

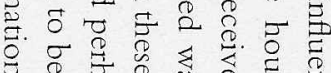

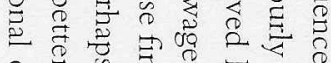
养:

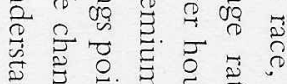

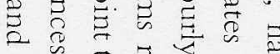

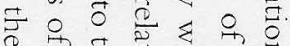

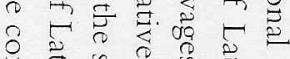

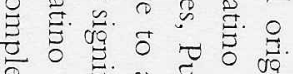

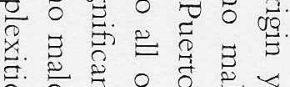

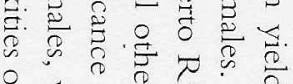

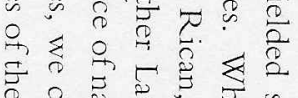

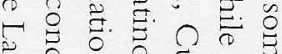
引.

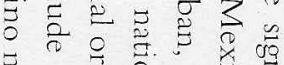

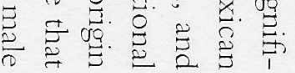



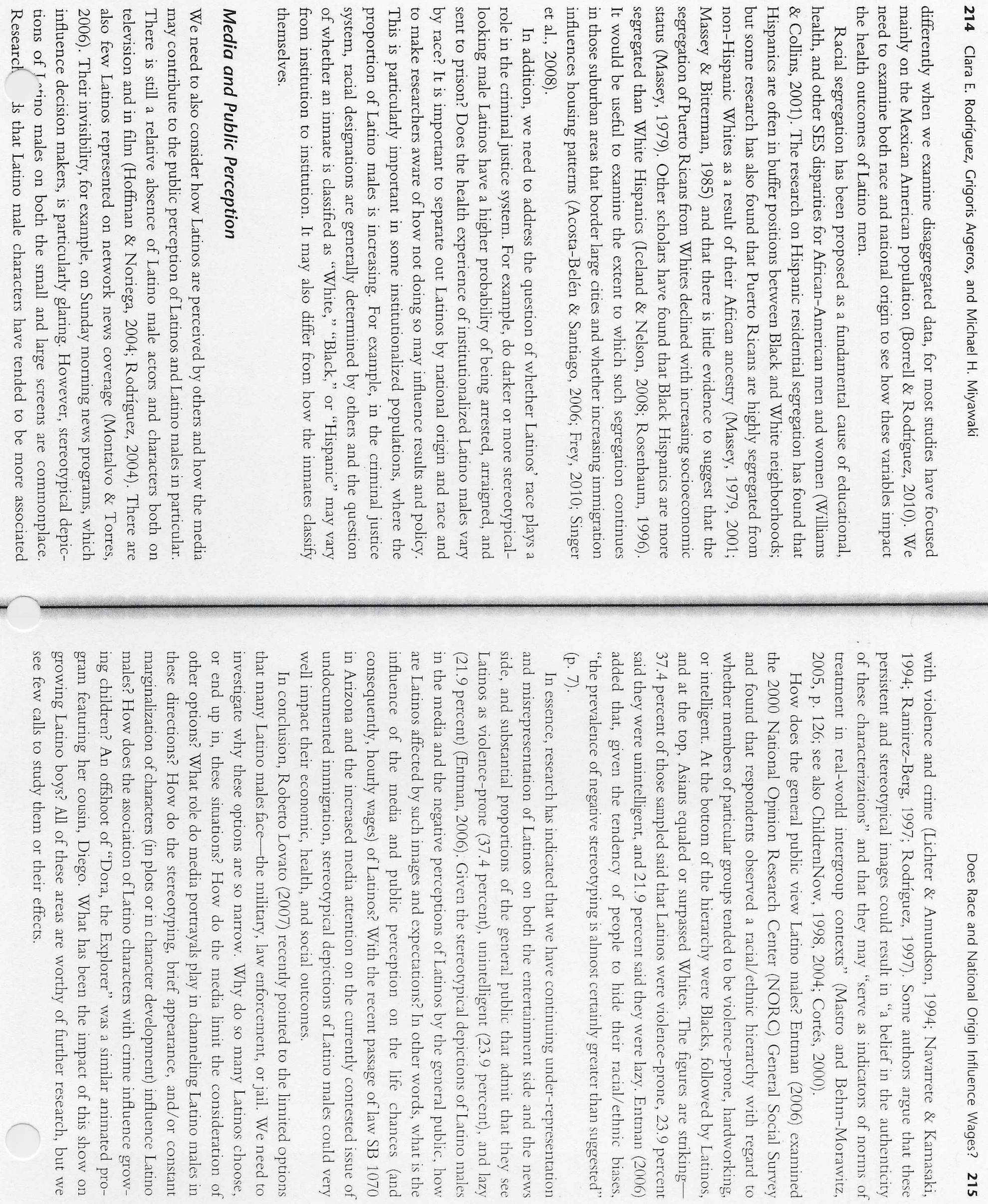


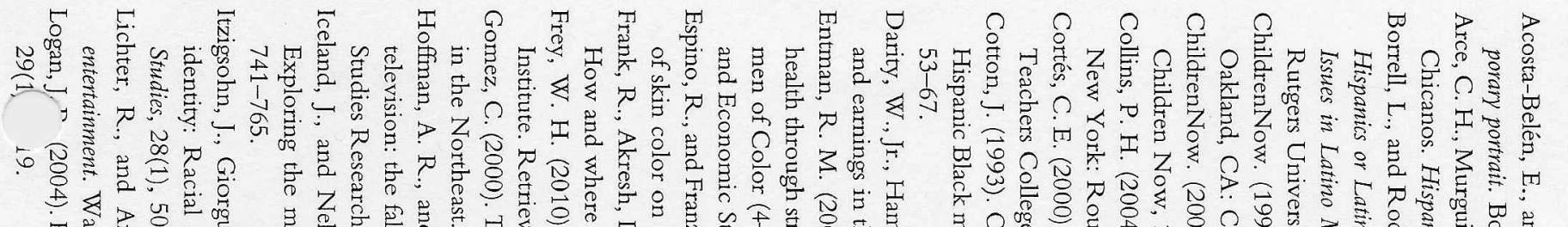

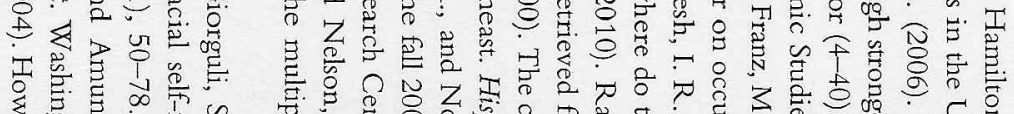
Wow

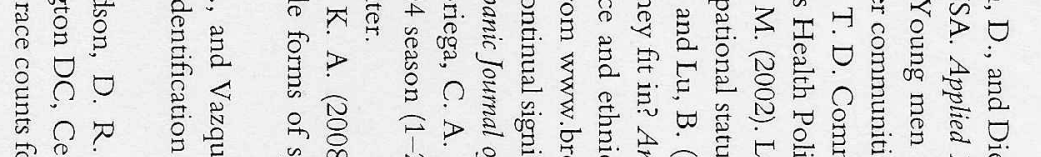

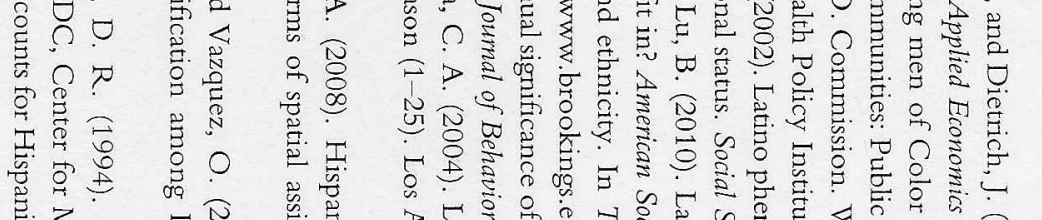

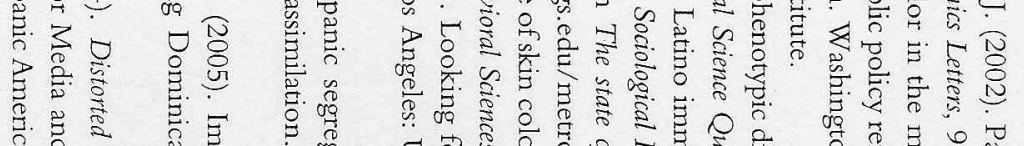

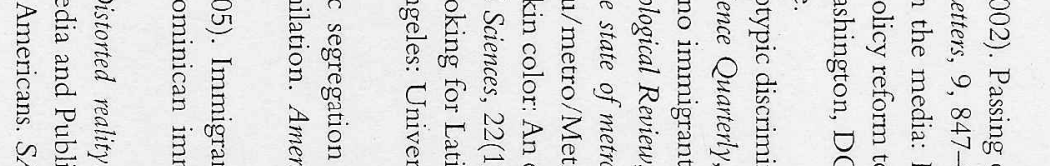

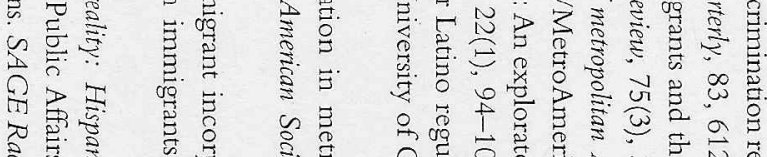

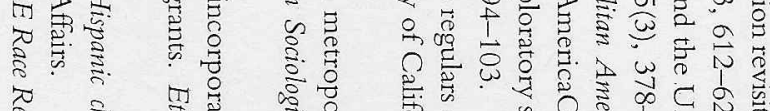
Pun

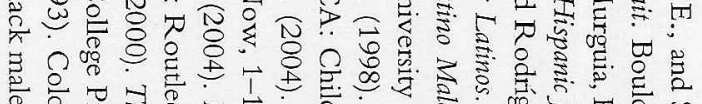

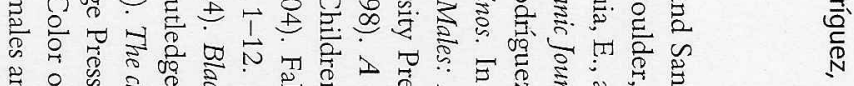

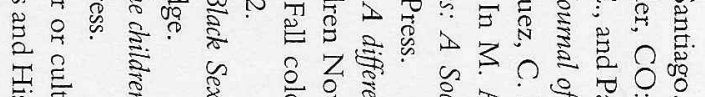

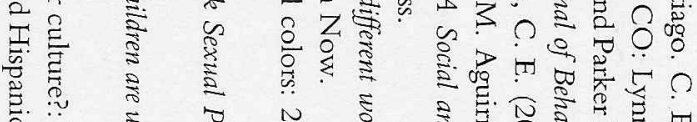

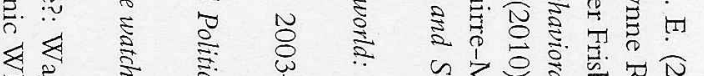

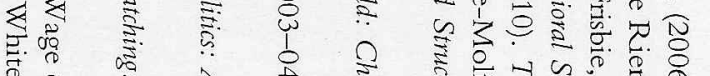

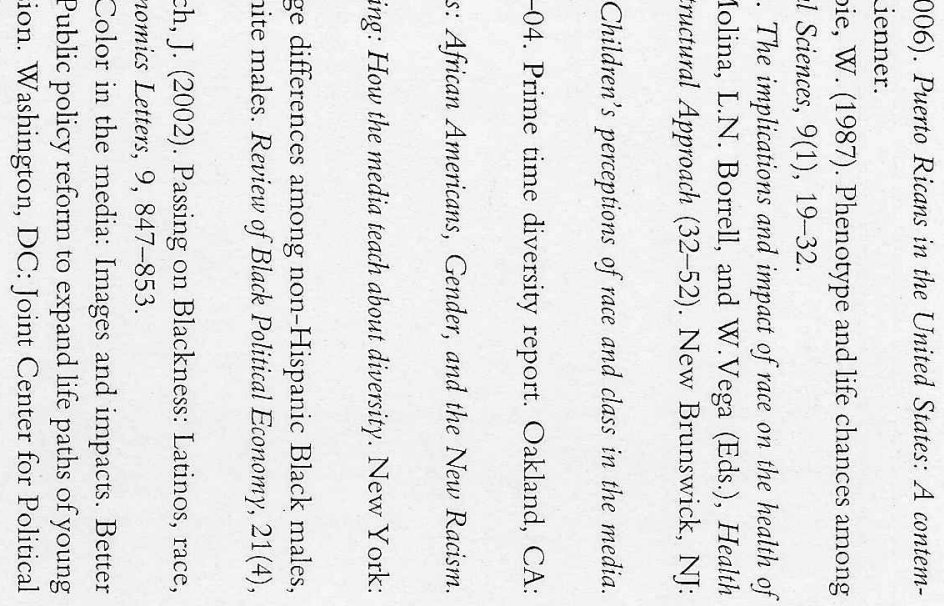
$\stackrel{\frac{0}{0}}{\frac{1}{0}} \stackrel{a}{a}$

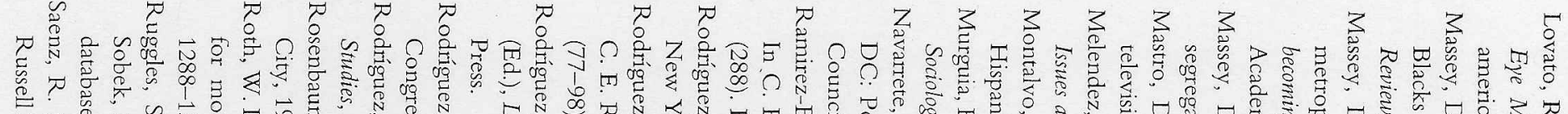

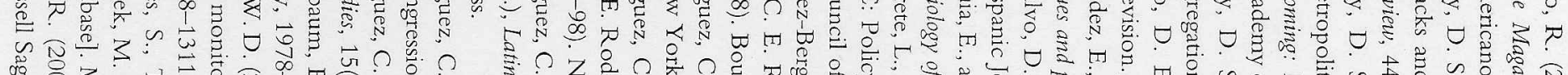
क

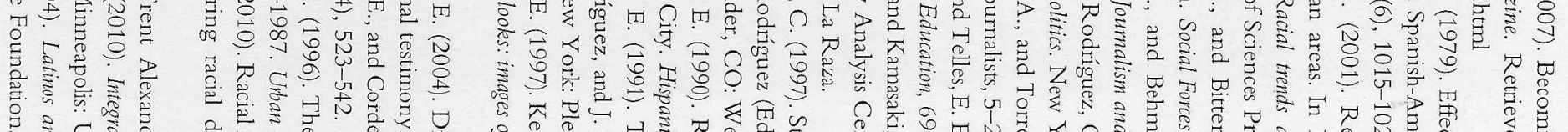

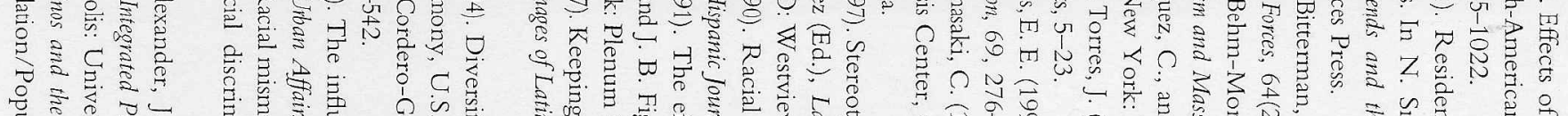

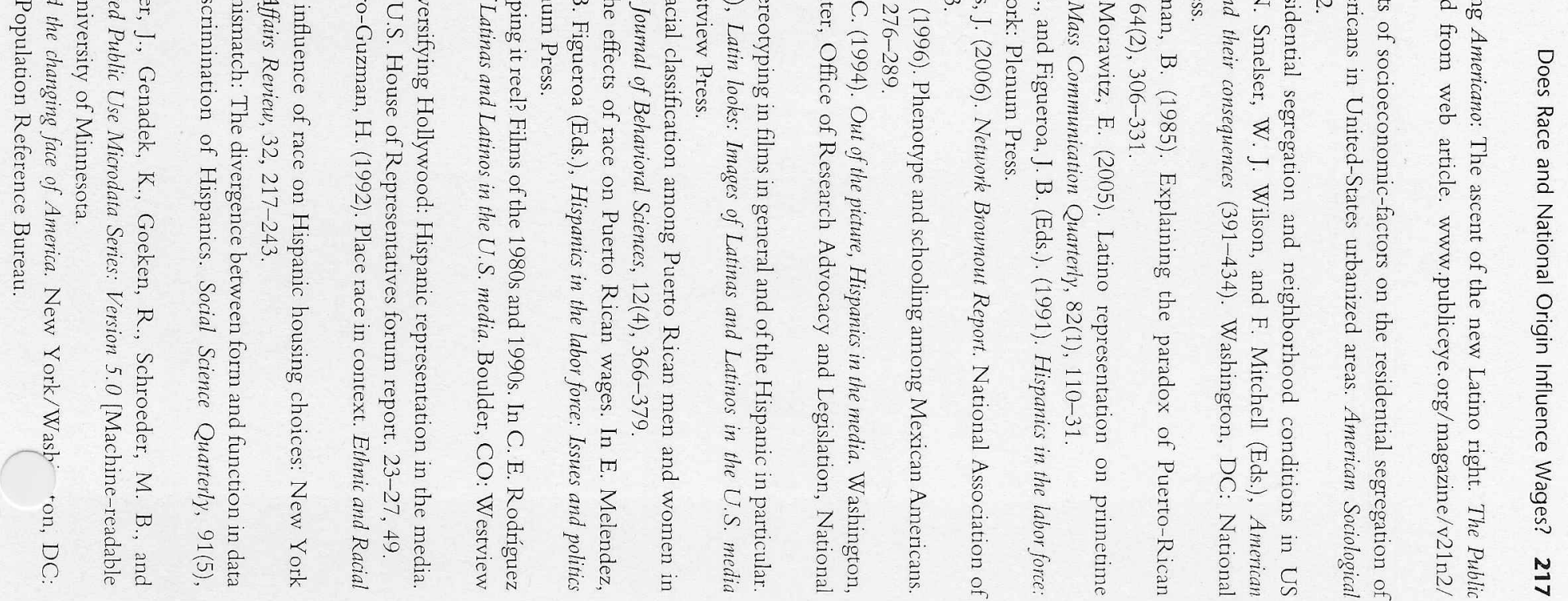



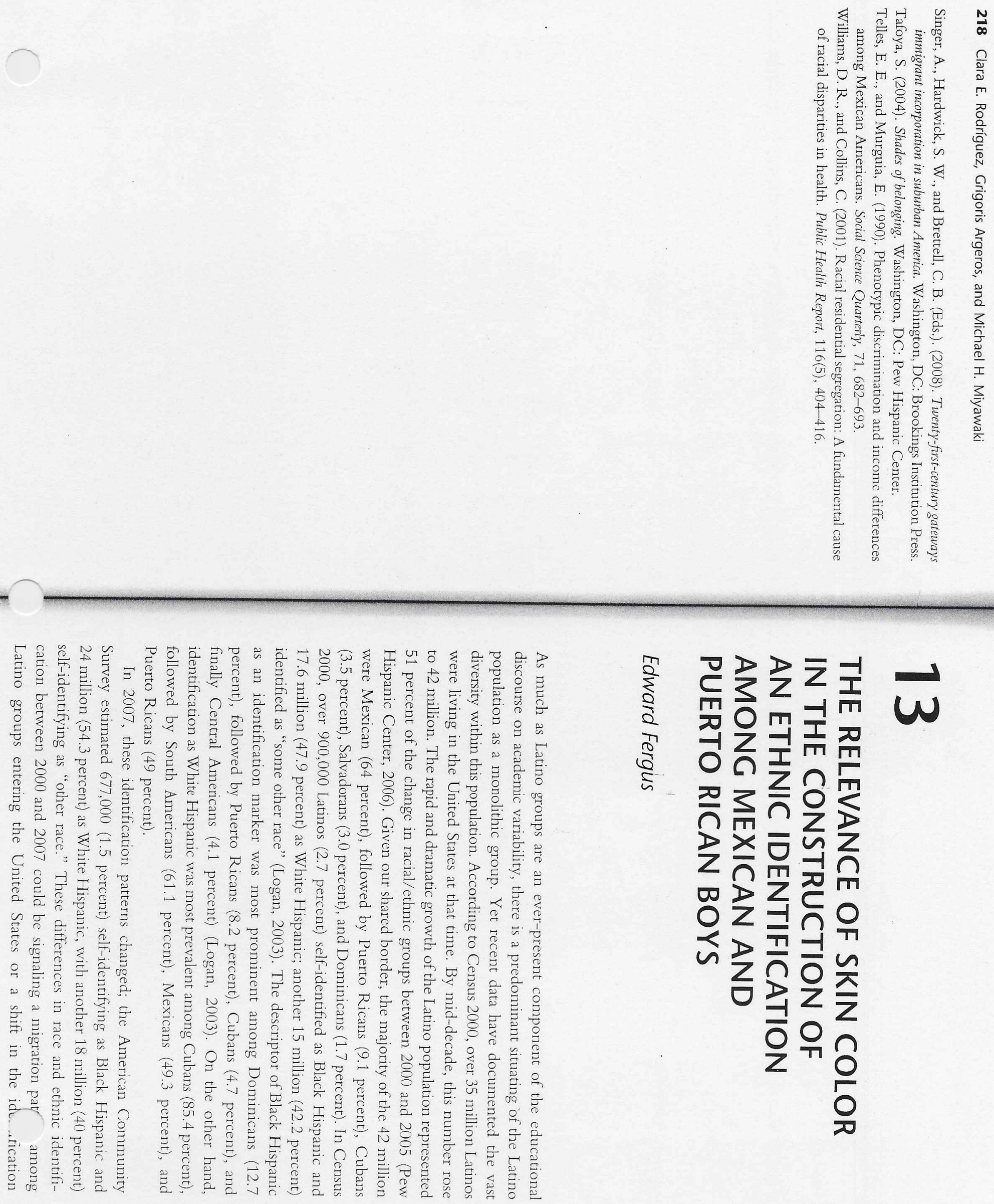


\section{EDUCATION}

"This urgent book, masterfully compiled by Noguera, Hurtado and Fergus, paints a nuanced portrait of the lives of the men and boys of America's fastest growing ethnicity is an indispensable book, children will inherit."

Latinolo Suárez-Orozeo is the Ross University Professor at New York University and co-author of

"Essential reading for anyone seeking to pierce the veil that distorts and obscures the realities of Latino men and boys. Impressive in scope, ranging from education opportunities, to homophobia, to the loneliness that attends boys' passage into manhood. Excellent and bracing and important."

Junot Diaz is the author of Drown and The Brief Wondrous Life of Oscar Wao

Latino men and boys in the United States are confronted with a wide variety of hardships that are not easily explained or understood. They are populating prisons, dropping out of high school, are becoming overrepresented in the service industry at alarming degrees. Young Latino men, especlally, have among the lowest wages mortality rates dunty, a rapidly srowing rate. of HIV/AIDS, and one of the highest status of men in Am herricing interest in the work avallable on Latino men and boys.

This groundbreaking interdisciplinary volume, edited by renowned scholars Pedro Noguera, Aida Hurtado and Edward A. Fergus, addresses the dearth of scholarship unique challenges ant Latino men and boys to further our understanding of the The contributors represent aces that they confront during this historical moment. Justice, education, literature, poss section of disciplines from health, criminal drawing attention to the sweeplinology, economics, labor, sociology and more. By volume offers research and poligy lssues facing this segment of the population, this decreasing the invisibility and thus the of principles and overarching guidelines for

Pedro Noguera is Peter L. Agnew Professor of Education in thr Culture, Education, and Hum

Aida Hurtado is Professor and Luis Leal Endowed Chair in the Chicana and Chicano Studies Department at University of California, Santa Barbara.

Zdward Fergus is Deputy Director of the Metropolitan Center for Urban Education at New York University.

Routledge

Taylor \& Francis Group wururoutledge.com

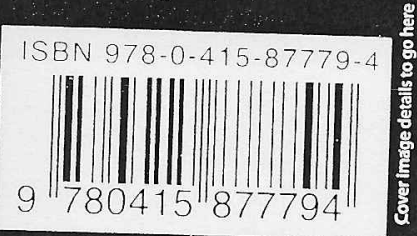

an liforma business 